\title{
Mechanical Characterization of Sheet Moulding Composites for the Automotive Industry
}

\author{
G. Lamanna* and A. Ceparano \\ Department of Industrial and Information Engineering, Second University of Naples, via Roma n. 29 - 81031 Aversa, \\ Italy
}

\begin{abstract}
The mechanical properties of Sheet Moulding Composite (SMC) have been analyzed by means of static and fatigue tests in tension. SMCs show a substantial in-plane anisotropy either in terms of stiffness or strength. Fatigue data were modeled adopting a fatigue model with two parameters, represented by the cyclic number and the mean stress. The statistical implementation of such model was based on the hypothesis that the monotonic tensile strength follows a twoparameter Weibull distribution. The model has the potential to be predictive indicating that the fatigue characterization of a given laminate can be achieved with a minimum number of experimental tests. The reliability of such procedure and its applicability limits are discussed in the light of the model parameters obtained for different glass fiber reinforced composites.
\end{abstract}

Keywords: Anisotropy, fatigue, SMC, tensile strength distribution.

\section{INTRODUCTION}

SMC represents a mixture of the subsequent components: polymer resin, fiber reinforcement, inert fillers, pigments, catalysts, release agents, stabilizers, and thickeners exactly as other thermosets. SMC is a composite material that is being used in several industrial applications; thanks to its aesthetic and mechanical properties it can be employed in the manufacturing of many components in a wide range of industrial fields: building constructions, electrical/ electronics, transportation, aerospace, chemical engineering, and the marine industry. The chopped glass fibers, that compose SMC, have a length ranging from 25 to $50 \mathrm{~mm}$ put in a thermosetting resin matrix system. SMC is normally manufactured in a conveyor belt where it is processed to form a sandwich structure containing the chopped fibers, resin matrix, and functional fillers, unlike fiber reinforced thermoplastics such as fiber reinforced PP composites. Then a shape, at a temperature of $140-170{ }^{\circ} \mathrm{C}$, is used to press the SMC for 1-10 minutes depending on the thickness and sizes of the panels. In this paper we analyze the mechanical properties of SMC panels used as low cost structural components in the automotive industry [1,2].

SMCs have emerged as suitable engineering materials due to their attributes that are competitive with existing engineering materials, especially metals. The low weight and low cost together with mechanical characteristics allow to reach cost-efficient performances in many weight-critical applications in spite of a product cost impediments compared with traditional materials. However, composites require higher sampling for their characterization compared to

*Address correspondence to this author at the Department of Industrial and Information Engineering - Second University of Naples, via Roma no. 29 81031 Aversa, Italy; Tel: +39 0815010 419; Fax: +39 0815010 295; E-mail: giuseppe.lamanna@unina2.it metals, being multiphase and anisotropic materials. In particular SMCs may show a substantial in-plane anisotropy due to the prevalent alignment of the fibers along the direction of the conveyor belt. Thus, similarly to other thermosetting resins-based composites, the inherent structural in-plane anisotropy of SMCs may induce distortions of parts both when the chemical shrinkage phenomena occur during cross-linking reactions and when non symmetrical cooling gives rise to substantial residual stress accumulation upon the components that are released from the mould. For this reason the mechanical characterization was carried out by taking specimens oriented at different angles in respect to the direction of the conveyor belt. The SMCs under study may undergo a severe temperature variations since the SMC components are located adjacent the engine compartment. This general aspect has been already highlighted showing that the fatigue behaviour of polymer based materials is influenced by its viscoelastic nature [3-10]. For instance, in [10] it was shown that conditioning a sample in the vicinity of $\mathrm{Tg}$, resulted in a residual stresses relaxation, leads to higher fatigue life compared to the materials as manufactured. In all, SMCs are very complex materials resulting from compounding of resin, fibers, and fillers. In service, the residual material strength as well as the component reliability is the result of the accumulation of a diffuse damage having different origin and location, rather than the propagation of a single crack as in metals. Thus, the damage mechanisms occur at different dimensional scales while the possible in-plane anisotropy makes different microstructural specimens features, leading to a different mechanical response that requires the mechanical characterization to be carefully performed on statistical basis. Concerning the fatigue behaviour the definition of reliable allowables, in terms of structural strength, represents one of the most difficult goal during the design phase of a component made of polymer composite 
because it is affected by a large scatter in mechanical properties [11-18]. The SMCs under study may be exposed in service to hostile conditions, e.g. service temperature, $\mathrm{Tg}$ near glass transition, cyclic loadings, or aggressive environments [15-26]. In particular the viscoelastic behaviour of the polymer matrix and its long-term behaviour can be altered by the vicinity of the glass transition temperature of the polymer itself [27-37].

The complex scenario described so far requires a very expensive and lengthy test campaign. This manuscript has the aim to provide a preliminary mechanical characterization of the material under study as the basis for the exploration of the thermo-mechanical issues. The static strength data are modeled on the basis of a two parameters Weibull distribution while for the fatigue data a model widely used in literature for glass fiber reinforced composite was adopted $[9-12,14]$. Under fixed loading conditions, the number of cycles at which fatigue failure occurs can be predicted by such model. It is assumed that the residual strength undergoes a continuous decay, following a power law, for their damage law.

\section{EXPERIMENTAL}

The material under study consists of a compound of polyester resin, chopped glass fibers, and calcium carbonate with a nominal wt/wt ratio of $40: 30: 30$, respectively. It is used as a structural components, at a low cost, in the automotive industry. However resin, fiber, and filler contents were checked by means of standard method DIN-EN-ISO11667. The results on five different samples showed that the percentage wt/wt of resin, fiberglass, and filler was 39:27:34, respectively. Samples were cut from sheets with nominal thickness $\mathrm{t}=2.5 \mathrm{~mm}$. The static tests were performed with a Zwick-Roell ${ }^{\mathbb{B}}$ Z010 dynamometer equipped with load cell of $10 \mathrm{kN}$. The deformations were measured with a Zwick extensometer with a gage length of $25 \mathrm{~mm}$. The data were processed with the Test Expert software by Zwick-Roell ${ }^{\mathbb{B}}$. The crosshead speed was $3 \mathrm{~mm} / \mathrm{min}$, according to ASTM D5083. The fatigue data in tension were performed at a frequency of $5 \mathrm{~Hz}$ on a Instron ${ }^{\mathbb{B}} 8100$ hydraulic machine.

\section{RESULTS AND DISCUSSION}

In Fig. (1) the results of static tests are reported. The relevant parameters are reported in Table $\mathbf{1}$ and expressed in terms of elastic modulus E, apparent yield stress $\sigma_{\mathrm{y}}$, maximum stress $\sigma_{\max }$, ultimate stress $\sigma_{b}$, deformation at maximum stress $\varepsilon\left(\sigma_{\max }\right)$, deformation at break $\varepsilon_{\mathrm{b}}$, together with the average values and the standard deviation.

From Fig. (1) it appears that the elastic modulus and the strength are correlated, confirming that fibers are not randomly oriented. To verify such hypothesis in Fig. (2) the nominal yield stress is reported as function of the elastic modulus. In the same figure the broken line represents the theoretical value of the elastic modulus corresponding to the lower bound (namely, when the fibers are oriented orthogonally to the applied load) and to the case of fibers with random distribution. The same approach is adopted in Fig. (3) where the cumulative distribution function of the elastic modulus is reported according to the Weibull equation; the general expression is:
$F(X \leq P)=1-\exp \left(\frac{\mathrm{P}}{\mathrm{b}}\right)^{\mathrm{k}}$

where $P$ is the generic property under study $b$ is its characteristic value and $k$ is a measure of the distribution spreading.

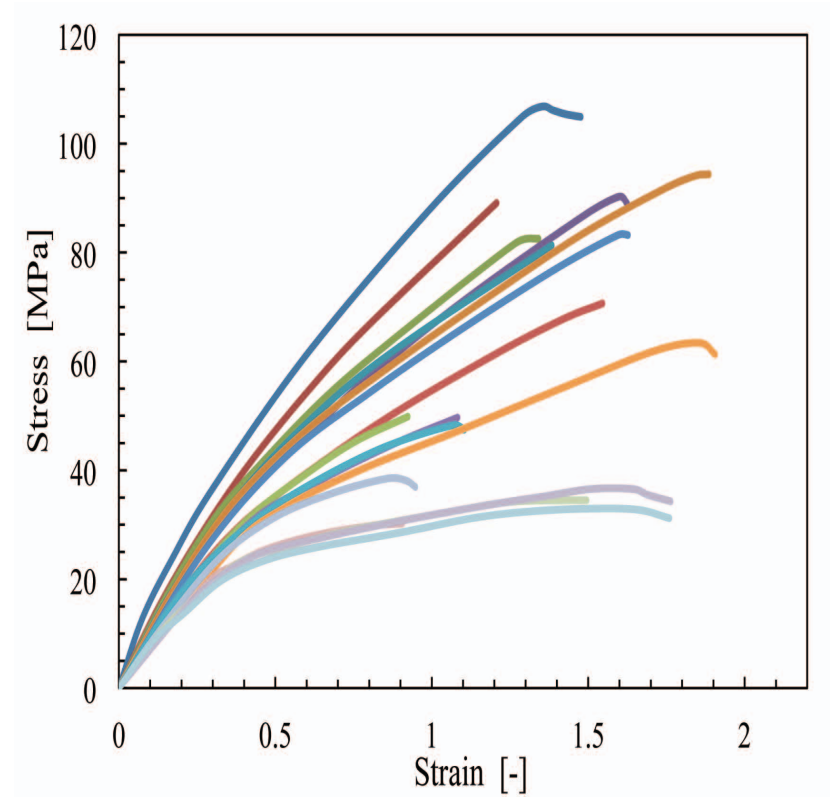

Fig. (1). Stress-strain curve at different sample orientation with respect to the conveyor belt direction. The crosshead speed is $3 \mathrm{~mm} / \mathrm{min}$ and the extensometer gauge length is $25 \mathrm{~mm}$.

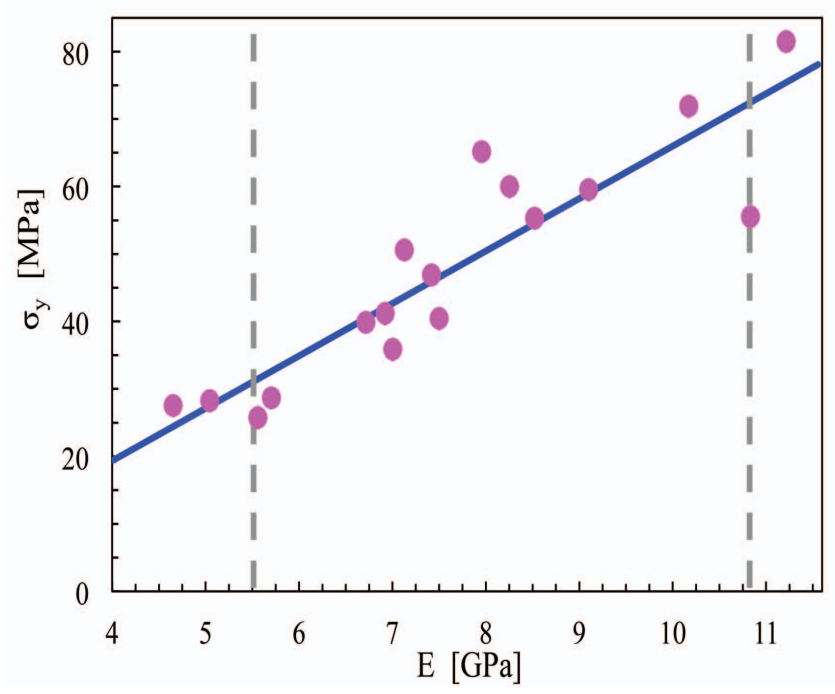

Fig. (2). The nominal yield stress $\sigma_{\mathrm{y}}$, as function of the elastic modulus E. Symbols represent the experimental data. The continuous line is a linear regression between of data. The broken lines represent the lower bound modulus (left) and the modulus of randomly distributed fibers.

Figs. $(3,4)$, where the distribution of $\sigma_{y}$ is reported (please, notice that $\sigma_{\mathrm{y}}$ represents a substantial measure of the elastic limit) confirm that in most of the samples the elastic modulus is bounded between the lower bound and the value attained by the modulus in case of fibers randomly 
Table 1. Summary of the results coming from stress-strain curves.

\begin{tabular}{|c|c|c|c|c|c|c|}
\hline & E/GPa & $\sigma_{\mathbf{y}} / \mathbf{M P a}$ & $\sigma_{\max } / \mathbf{M P a}$ & $\varepsilon\left(\sigma_{\max }\right) / \%$ & $\sigma_{\mathbf{b}} / \mathbf{M P a}$ & $\varepsilon_{b} / \mathbf{M P a}$ \\
\hline & 4.6 & 27.3 & 36.7 & 1.7 & 35.1 & 1.9 \\
\hline & 5.0 & 28.0 & 34.9 & 1.6 & 33.7 & 1.6 \\
\hline & 5.6 & 25.8 & 33.0 & 1.6 & 31.9 & 1.8 \\
\hline & 5.7 & 28.5 & 30.3 & 0.9 & 30.1 & 1.0 \\
\hline & 6.7 & 39.6 & 63.3 & 1.9 & 61.3 & 1.9 \\
\hline & 6.9 & 41.0 & 49.6 & 1.1 & 49.6 & 1.1 \\
\hline & 7.0 & 35.7 & 39.0 & 0.9 & 37.8 & 1.0 \\
\hline & 7.1 & 50.4 & 70.5 & 1.6 & 69.1 & 1.6 \\
\hline & 7.4 & 46.8 & 50.0 & 1.0 & 40.4 & 0.8 \\
\hline & 7.5 & 40.3 & 48.6 & 1.1 & 45.6 & 1.1 \\
\hline & 7.9 & 65.0 & 81.2 & 1.5 & 81.2 & 1.5 \\
\hline & 8.2 & 59.8 & 94.3 & 1.9 & 94.3 & 1.9 \\
\hline & 8.5 & 55.2 & 83.5 & 1.6 & 83.3 & 1.6 \\
\hline & 9.1 & 59.4 & 90.0 & 1.6 & 89.1 & 1.6 \\
\hline & 10.2 & 71.8 & 89.2 & 1.2 & 89.1 & 1.2 \\
\hline & 10.8 & 55.5 & 82.5 & 1.4 & 82.5 & 1.4 \\
\hline & 11.2 & 81.2 & 106.7 & 1.4 & 105.2 & 1.5 \\
\hline average & 7.6 & 47.7 & 63.7 & 1.4 & 62.3 & 1.4 \\
\hline St. dev. & 1.9 & 16.5 & 25.1 & 0.3 & 25.7 & 0.3 \\
\hline
\end{tabular}

distributed. This is a clear manifestation of the in-plane anisotropy.

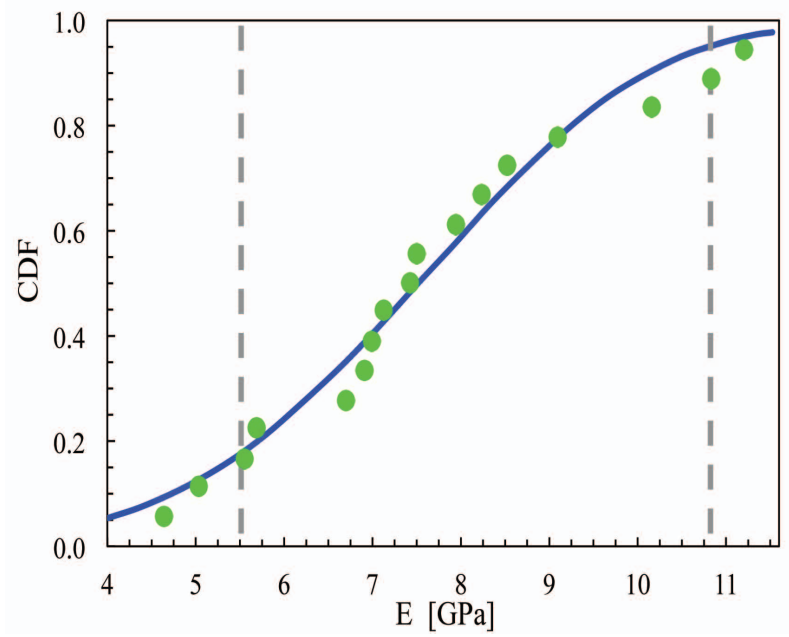

Fig. (3). Cumulative distribution function for the elastic modulus. Symbols represent the experimental data, continuous line the Weibull fit with $\mathrm{b}=8.22054$ and $\mathrm{k}=4.07054$. Broken lines, as defined in Fig. (2).

Looking again at Fig. (1), one can recognize three main responses having low, medium, and high modulus, respectively. Accordingly, the statistical analysis for these three distinct behaviours is reported in Figs. (5-8) for the elastic modulus, the elastic limit, and the stress at break.

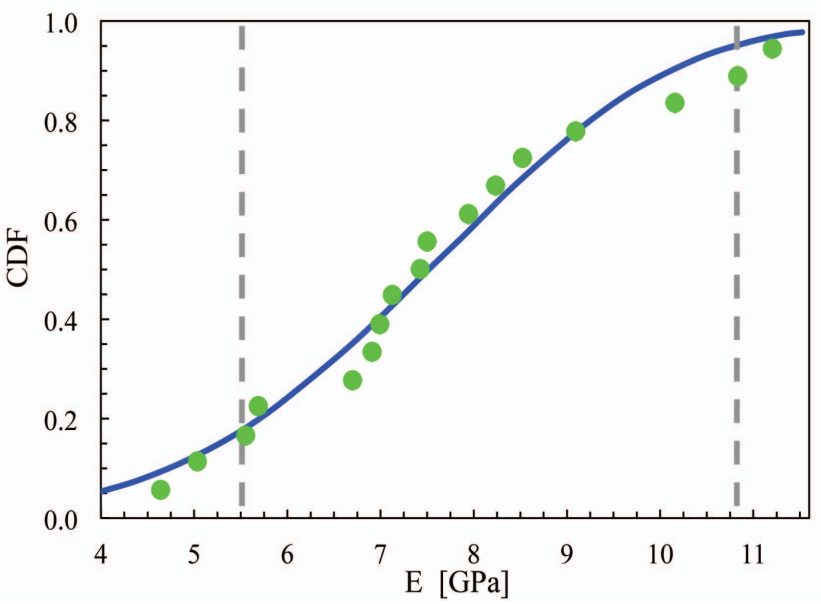

Fig. (4). Cumulative distribution function for the elastic limit, $\sigma_{y}$. Symbols: experimental data, the continuous line is the Weibull fit with $\mathrm{b}=53.5554$ and $\mathrm{k}=2.72365$.

The S-N cyclic fatigue data are reported in terms of the normalized maximum stress $\sigma_{\max } / \sigma_{0},\left(\sigma_{0}\right.$ is the static material strength), the maximum cyclic stress, $\sigma$ max, or stress amplitude $\sigma_{\max }-\sigma_{\min }$, as a function of number of cycles to failure N. In this paper the value of the maximum stress is fixed with the cyclic load determined by the stress ratio 


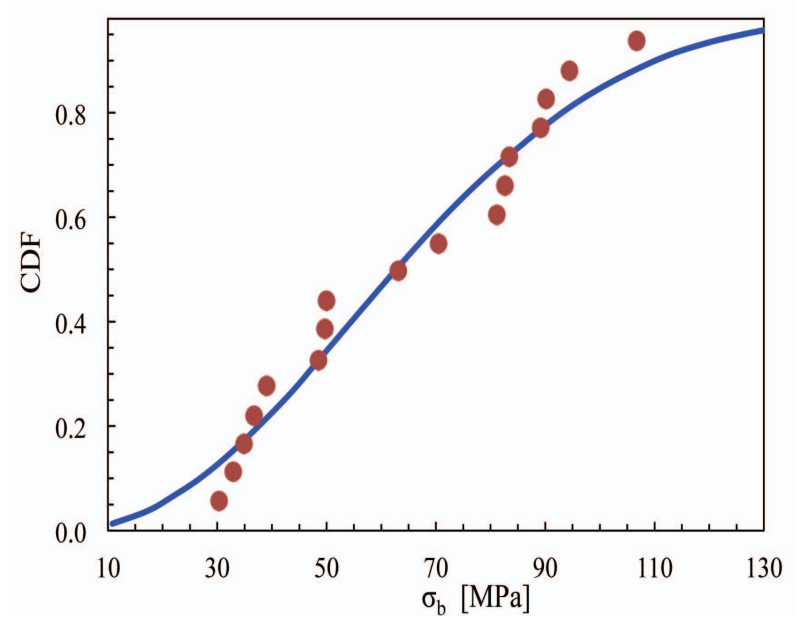

Fig. (5). Cumulative distribution function for the stress at break $\sigma_{b}$. Symbols: experimental data; continuous line: Weibull fit with $\mathrm{b}=73.5306, \mathrm{k}=2.16924$.

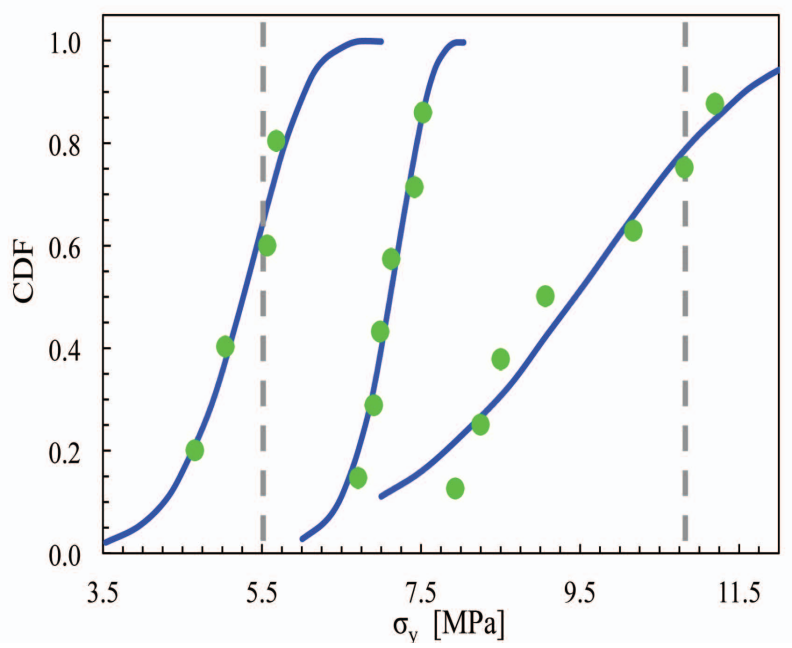

Fig. (6). Cumulative distribution of the elastic modulus. Symbols: experimental data; the continuous lines are the curve fitting with the Weibull distribution. The parameters of the distribution functions are (from left to the right): $b_{1}=28.0177$ and $k_{1}=21.2876$; $\mathrm{b}_{2}=44.7353$ and $\mathrm{k}_{2}=6.386 ; \mathrm{b}_{3}=67.2306$ and $\mathrm{k}_{3}=5.98318$.

$\mathrm{R}=\sigma_{\min } / \sigma_{\max }=0.1$ (a loading condition commonly used for polymer composites) and then all the fatigue data are found for such fixed value. The maximum stress, $\sigma_{\max }$, is chosen as a percentage of the characteristic strength $\sigma_{\text {mean }}=53 \mathrm{MPa}$. Cyclic loading was performed with a triangular waveform with the loading - unloading rate of $3 \mathrm{~mm} / \mathrm{min}$. Two loading conditions have been selected in terms of normalized maximum stress, namely $\sigma_{\min } / \sigma_{\max }=0.8$ and $\sigma_{\min } / \sigma_{\max }=0.7$. However, given $\sigma_{\max }$, higher stress ratios, $\mathrm{R}$, imply higher cycles to failure. Therefore to fit the experimental data we adopt a phenomenological analytic model appeared in literature that assumes $\mathrm{R}$ as the main variable $[9-12,14]$.

Furthermore, the concept of "strength-life equal rank assumption" is well established in polymer composites as it

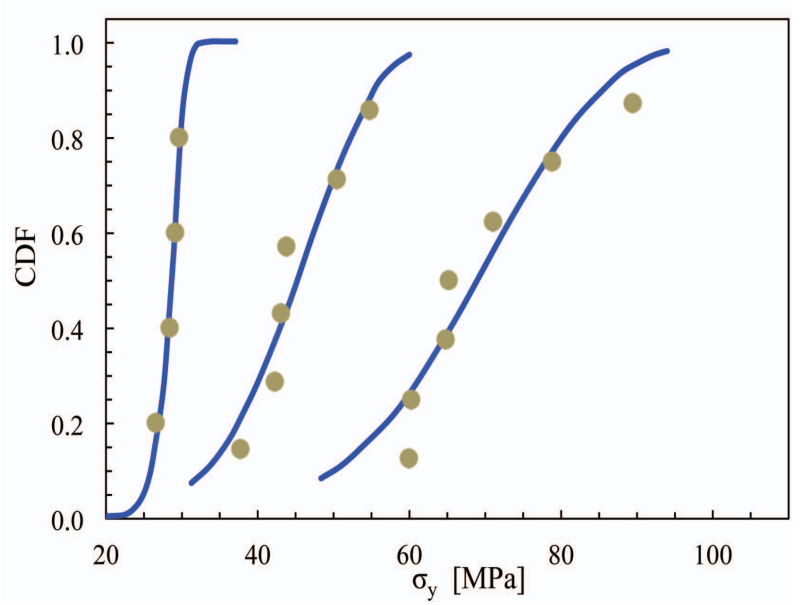

Fig. (7). Cumulative distribution of the elastic limit $\sigma_{y}$. Symbols: experimental data; the continuous lines are the curve fitting with the Weibull distribution. The parameters of the distribution function are (from left to the right): $\mathrm{b}_{1}=28.0177$ and $\mathrm{k}_{1}=21.2876 ; \mathrm{b}_{2}=44.7353$ and $\mathrm{k}_{2}=6.386 ; \mathrm{b}_{3}=67.2306$ and $\mathrm{k}_{3}=5.98318$.

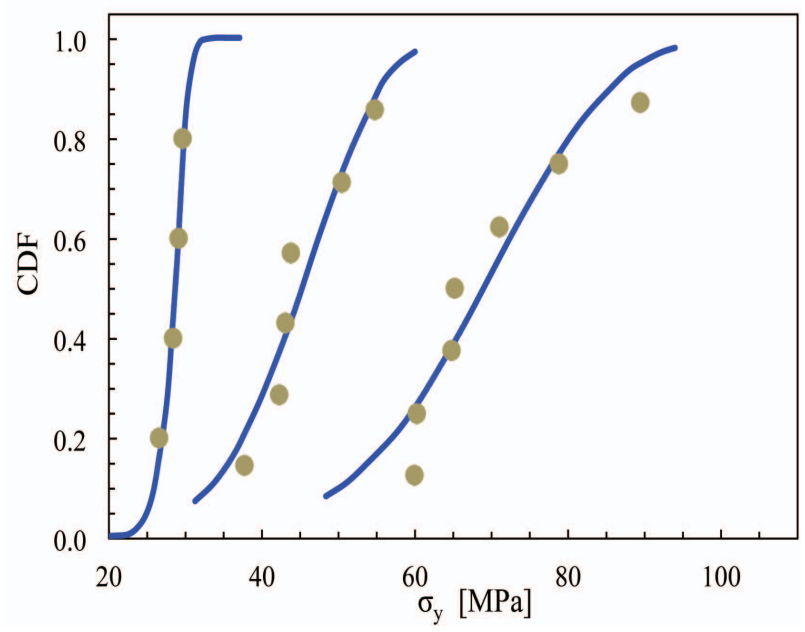

Fig. (8). Cumulative distribution of the stress at break $\sigma_{b}$. Symbols: experimental data; the continuous lines are the curve fitting with the Weibull distribution. The parameters of the distribution function are (from left to the right): $\mathrm{b}_{1}=35.1212, \mathrm{k}_{1}=10.5269 ; \mathrm{b}_{2}=58.5418$, $\mathrm{k}_{2}=3.78681 ; \mathrm{b}_{3}=91.258, \mathrm{k}_{3}=12.0063$.

reflects the fact that the dispersion of fatigue data is a mirror image of the dispersion of static strength data, provided a statistical ensemble of static and dynamic data are available.

The phenomenological model under study was used in different fiberglass reinforced composites with thermoplastic or thermosetting matrices. The model has the potential to be implemented statistically and assumes the following form [10]

$\frac{\sigma_{\max }}{\sigma_{\text {mean }}}=\left[\alpha\left(\mathrm{N}^{\beta}-1\right)(1-\mathrm{R})+1\right]^{-1}$

where $\alpha$ and $\beta$ are the model parameters, $N$ is the number of cycles to failure, and $\mathrm{R}$ has been already defined. Finally, an 
interesting form of the model is taken into account as it allows recovering the statistical strength of virgin samples subjected to fatigue loads.

$\sigma_{0}=\sigma_{0 N}=\sigma_{\max }\left[\alpha\left(N^{\beta}-1\right)(1-R)+1\right]$

In Equation 3, from the fatigue data taken at different maximum stress can be deduced the distribution of the static strength, $\sigma_{0 \mathrm{~N}}$, of the virgin material.

In Fig. (9) the fatigue data are reported. The data are obtained fixing the maximum stress to the mean strength ratio, $\sigma_{\max } / \sigma_{0 \text { mean }}$, where $\sigma_{0 \text { mean }}$ is the mean static strength obtained describing the static strength data with a two parameters Weibulll distribution. Then, given the loading ratio $\mathrm{R}=\sigma_{\min } / \sigma_{\max }$, the stress amplitude $\Delta \sigma=\sigma_{\max }-\sigma_{\min }$ is fixed. In order to accelerate the response in fatigue two levels of maximum stress were adopted, such that $\left(\sigma_{\max } / \sigma_{0 \text { mean }}\right)=0.8$ and 0.6. The experimental data are then elaborated by a best fitting procedure to obtain the parameters of Equation 2. To summarize, in Table 2 are reported the model parameters for the SMCs under study together with those found in literature on different fiberglass composites.

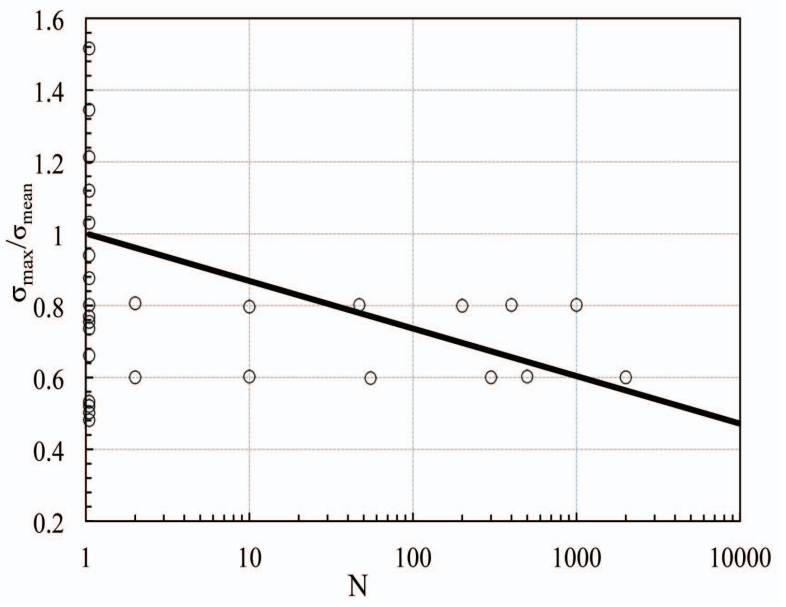

Fig. (9). The normalized maximum stress as function of cycle to rupture. The symbols represent the experimental data; continuous line: best fitting according to Equation 2 .

Furthermore, using Equation 3 one can recover the static strength of samples tested in fatigue. This procedure is a way to test the reliability of the model parameters. The strength, $\sigma_{0 \mathrm{~N}}$, of the virgin samples tested in fatigue are unknown, obviously. However the adopted theory [10] allows to recover such values directly from fatigue data, provided that $\alpha$ and $\beta$ are reliable parameters. Thus, in principle, the distribution of the static strength coming from fatigue data, $\sigma_{0 \mathrm{~N}}$, should superpose to the distribution of static strength data. In both cases a two parameters Weibull distribution can be adopted. The results of this procedure are illustrated in Fig. (10) and can be summarized as follows: the mean strength of the $\sigma_{0 \mathrm{~N}}$ distribution coming from dynamic tests is $\sigma_{0 \text { Nmean }}=57 \mathrm{MPa}$ while the static strength counterpart was $\sigma_{0 \text { mean }}=53 \mathrm{MPa}$. Instead, the shape factors differ much more (see the numerical values of mean strength and shape factor of Table 2), and this is a sign of the limited number of fatigue tests under study. To this end, we argue that the optimum number of fatigue tests to obtain reliable $\alpha$ and $\beta$ parameters of Equation 2 is strictly linked to the shape factor of the Weibull distribution of the static strength. In fact, the superposition of static strength distribution, $\sigma_{0}$, to the strength distribution coming out from fatigue data, $\sigma_{0 \mathrm{~N}}$, is readily obtained on materials showing narrower distribution of the static strength as shown in $[10,11,14]$. Nonetheless, the approach appears as a promising guideline to study the thermo-mechanical behaviour of SMCs, when deeper statistical analysis is needed.

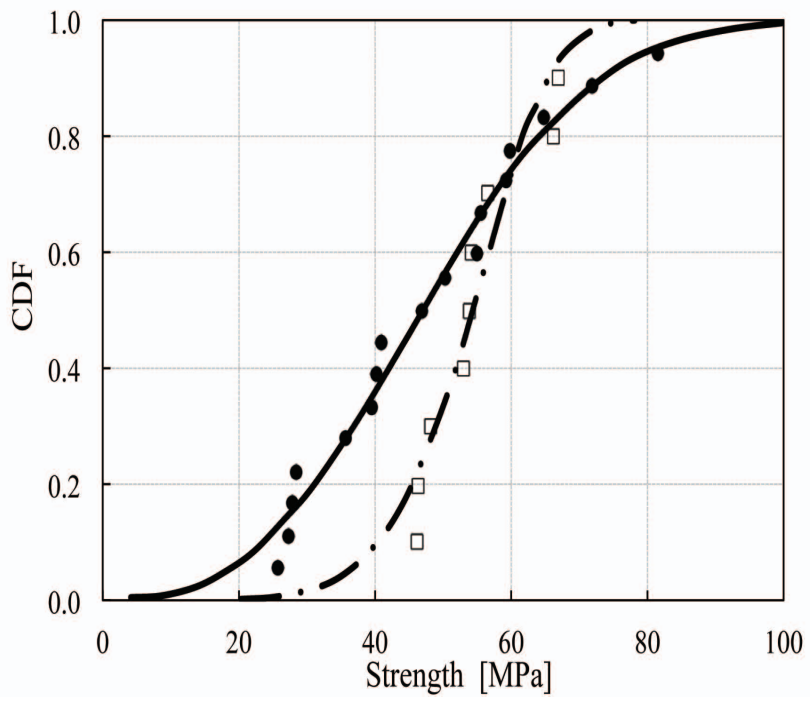

Fig. (10). Statistical distribution of static strength $\sigma_{0}$ (filled symbol) and the calculated (according to Equation 3) monotonic strength, $\sigma_{0 \mathrm{~N}}$, for SMC under study.

Table 2. Comparison of fatigue and Weibull model parameters for different fiberglass reinforced composites.

\begin{tabular}{|c|c|c|c|c|c|c|}
\hline Material & $\mathbf{v}_{\mathbf{f}}$ & $\boldsymbol{\sigma}_{\mathbf{R}}(\mathbf{M P a})$ & $\boldsymbol{\alpha}$ & $\boldsymbol{\beta}$ & $\boldsymbol{\gamma}(\mathbf{M P a})$ & $\boldsymbol{\delta}$ \\
\hline \hline TSC & 0.35 & 290 & 0.353 & 0.150 & 298 & 20.31 \\
\hline SMC & 0.22 (nom) & 53 & 0.250 & 0.168 & 53.55 & 2.72 \\
\hline TPC & 0.20 & 185 & 0.224 & 0.194 & 195 & 11.93 \\
\hline TPP & 0.16 & 146 & 0.135 & 0.236 & 154 & 10.34 \\
\hline
\end{tabular}

$v_{\mathrm{f}}$ : Volume fraction of glass fibers; $\sigma_{\mathrm{R}}:$ Average static strength; $\alpha, \beta$ : fatigue model parameters; $\gamma$ : shape parameter of Weibull distribution of static strength; $\delta=$ mean value of Weibull distribution of static strength.

$\mathrm{TSC}=$ ThermoSetting Continuous fibers; $\mathrm{SMC}=$ Sheet Moulding Composite; $\mathrm{TPC}=$ ThermoPlastic Continuous fibers; $\mathrm{TPP}=\mathrm{ThermoPlastic}$ Particulate-short fibers. 


\section{CONCLUSION}

SMCs are very complex materials showing a substantial anisotropy due to the preferential orientation of chopped fibers along the conveyor belt. The selection of their properties at the design stage is not easy when a deep mechanical characterization is not considered. The fatigue behaviour of such materials is in line with the trend showed by other materials, namely it depends substantially on the volume fraction of fibers.

\section{CONFLICT OF INTEREST}

The authors confirm that this article content has no conflicts of interest.

\section{ACKNOWLEDGEMENTS}

Declared none.

\section{REFERENCES}

[1] Garmendia J, Olaizola M, Etxeberria I, Franco JC, Mondragon I. Influence of processing and moulding conditions on the mechanical behavior of sheet-moulding compound laminates. J Mater Sci 1995; 30: 5287-94.

[2] Zouhaier J, Fodil M, Joseph F, Didier B. Multi-scales modelling of dynamic behaviour for discontinuous fiber SMC composites. Compos Sci Technol 2009; 69(1): 97-103.

[3] Grassia L, D'Amore A. Modeling the residual stresses in reactive resins-based materials: A case study of photo-sensitive composites for dental applications. AIP Conf Proc 2010; 1255: 408-10.

[4] Grassia L, D'Amore A. Calculation of the shrinkage-induced residual stress in a viscoelastic dental restorative material. Mech Time-Depend Mater 2013; 17(1): 1-13.

[5] D'Amore A, Caputo F, Grassia L, Zarrelli M. Numerical Evaluation of Structural Relaxation-Induced Stresses in Amorphous Polymers. Compos Part A-Appl S 2006; 37: 556-64.

[6] Grassia L, D'Amore A. Residual stresses in amorphous polymers. Macromol Symp 2005; 228: 1-15.

[7] Zarrelli M, Partridge IK, D'Amore A. Warpage induced in bimaterial specimens: Coefficient of thermal expansion, chemical shrinkage and viscoelastic modulus evolution during cure. Compos Part A-Appl S 2006; 37: 565-70.

[8] Yang Y, D'Amore A, Di Y, Nicolais L, Li B. Effect of physical aging on phenolphthalein polyethersulfone/poly(phenylene sulfide) blend. I. Mechanical properties. J Appl Polym Sci 1996; 59: 115966.

[9] D'Amore A, Caprino G, Nicolais L, Marino G. Long-term behaviour of PEI and PEI-based composites subjected to physical aging. Compos Sci Technol 1999; 59: 1993-2003.

[10] D'Amore A, Caprino G, Stupak P, Zhou J, Nicolais L. Effect of stress ratio on the flexural fatigue behaviour of continuous strand mat reinforced plastics. Sci Eng Compos Mater 1996; 5: 1-8.

[11] D'Amore A, Verde P. Modeling the flexural fatigue behavior of glass-fiber-reinforced thermoplastic matrices. Mech Time-Depend Mater 2013; 17(1): 15-23.

[12] D'Amore A, Grassia L, Verde P. Modeling the Fatigue Behavior of Glass Fiber Reinforced Thermoplastic and Thermosetting Matrices. AIP Conf Proc 2012; 1459: 372-4.

[13] Fawaz Z, Ellyn F. Fatigue Failure Model for Fibre-Reinforced Materials under General Loading Conditions. J Compos Mater $1994 ; 28(15): 1432-51$.
[14] Caprino G, D'Amore A. Flexural fatigue behaviour of randomcontinous-fiber-reinforced thermoplastic composites. Compos Sci Technol 1998; 58(6): 957-65.

[15] Hahn HT, Kim RY. Proof testing of composite materials. J Compos Mater 1975; 9: 297-311.

[16] Chou PC, Croman R. Residual Strength in Fatigue Based on Strength-life equal rank assumption. J Compos Mater 1978; 12: 177-94.

[17] Yang JN, Liu MD. Residual strength degradation model and theory of periodic proof tests for graphite/epoxy laminates. J Compos Mater 1977; 11: 176-203.

[18] Yang JN. Effect of high load on statistical fatigue of unnoched graphite/epoxy laminates. J Compos Mater 1980; 14: 82-94.

[19] Lamanna G, Ceparano A, Sartore L. Reliability of sheet moulding composites (SMC) for the automotive industry. AIP Conf Proc 2014; 1599: 339-42.

[20] Grassia L, D'Amore A, Verde P. On The Inter-Conversion Between Viscoelastic Material Functions of Polycarbonate. AIP Conf Proc 2012; 1459: 375-7.

[21] Martone A, Grassia L, Zarrelli M, Giordano M, D'Amore A Enthalpy relaxation of an epoxy matrix/carbon nanotubes. AIP Conf Proc 2012; 1459: 347-9.

[22] Lamanna G, Grassia L, Ascione V. Experimental investigation on the residual stresses induced by a plastic forming process. Key Eng Mat 2012; 452: 821-4.

[23] Annicchiarico A, Caputo F, De Angelis G, Frascà F, Lamanna G. Torsional stiffness verification of an adhesively bonded joint. AIP Conf Proc 2010; 1255: 398-401.

[24] Lamanna G, Basile A. Mechanics of soft PSAs (pressure sensitive adhesives). Open Mater Sci J 2013; 7: 23-8

[25] Alfani R, Colombet P, D'Amore A, Rizzo N, Nicolais L. Effect of temperature on thermo-mechanical properties of Macro-DefectFree cement-polymer composite. J Mater Sci 1999; 34: 5683-7.

[26] Netti P, D'Amore A, Ronca D, Ambrosio L, Nicolais L. Structuremechanical properties relationship of natural tendons and ligaments. J Mater Sci-Mater M 1996; 7: 525-30.

[27] Miyano Y, MacMurray MK, Enyama J, Nakada M. Loading rate and temperature dependence on flexural fatigue behavior of a satin woven CFRP laminate. J Compos Mater 1994; 28(13): 1250-60.

[28] Caputo F, Lamanna G, Soprano A. Crack tip parameters under Large Scale Yielding condition. Structural Durability \& Health Monitoring 2014; 2: 1-15.

[29] Lamanna G, Caputo F, Soprano A. Handling of composite-metal interface in a hybrid mechanical coupling. AIP Conf Proc 2012; 1459: 353-5.

[30] Grassia L, D'Amore A, Simon Sl. On the viscoelastic Poisson's ratio in amorphous polymers. J Rheol 2010; 54: 1009-22.

[31] Miyano Y, Nakada M, Kudok H, Muki R. Prediction of tensile fatigue of for unidirectional CRFP. J Compos Mater 2010; 34(7): 538-50.

[32] McCrum G, Bickley CP, Bucknall CB. In: Principles of Polymer Engineering; Oxford University Press 1989

[33] Caputo F, Lamanna G, Pannullo FM, De Angelis G. A methodological approach to the tolerance problems during the assembly process of deformable bodies. Key Eng Mat 2012; 488-9: 557-60.

[34] Chung I, Sun CT, Chang IY. Modeling creep in thermoplastic composites. J Compos Mater 1993; 27: 1009-29.

[35] Grassia L, D'Amore A. Isobaric PVT behavior of Poly(Carbonate) (PC). AIP Conf Proc 2010; 1255: 417-9.

[36] Grassia L, D'Amore A. Isobaric and isothermal glass transition of PMMA: Pressure-volume- temperature experiments and modelling predictions. J Non-Cryst Solids 2011; 357: 414-8.

[37] Grassia L, Pastore CMG, Mensitieri G, D'Amore A. Modeling of density evolution of PLA under ultra-high pressure/temperature histories. Polymer 2011; 52: 4011-20. 